\title{
IV. Botanik.
}

\section{Uebersicht der Gefass-Kryptogamen, Farnkrăuter, Fillees L., im Umfange von Reichenbachs Flora Germaniae excursoria.}

\author{
Von Dr. M. F. Löhr.
}

Blüthenlose Gefässpflanzen, Endogenae cryptogamicae seu Acotyledonae vasculares De Candolle, Koch Syn. edit. II. p. 963 .

A. Goniopterides Willd. Spec. pl. V. Calamaria Endl. Syst.

1. Ord. Equis etace en De Cand. Fl. Fr. 2. p. 580. Koch Syn. p. 963. Döll. rheinische Flora 1843. Schafthalme.

1) Equisetum Linné gen. pl. Koch. Syn. Döll. rh. Fl. p. 26. Schafthalm, Schaftheu.

1) E q. arvense Lin. spec. pl. K. Syn. Acker-Schafthalm: Döll. rh. Fl. Garke Deutschlands Flora. VI. Aufl. p. 467. Rabenhorst Krypt. Milde schles. Krypt. fig. 10-25. Schkur Kryptog. tab. 167. Aendert ab:

b) alpestre Wahlb. Lappon. p. 296. Auf Hochgebirgen und Voralpen durch die Alpenkette und auf den Karpathen in Siebenbürgen.

c) nemorosum Al. Braun in Döll. rh. Flora p. 27. Eq. arvense $\beta$. Schltz Starg. suppl. p. 58. Eq. pratense Roth tentam. III. (non Ehrh.) Sterile, langästige Form, stellw. auf feuchten sumpfigen Waldstellen, Wiesen.

d) boreale Ruprecht in Garke Deutschl. Flora VI. Aeste der unfruchtbaren Stengel 3 kantig mit 3 zähnigen Scheiden. So nur bis jetzt bei Königsberg beobachtet. Als wahrscheinlich zufällige Formen sind noch : 
e) irriguum Milde schles. Krypt. p. 421. fig. $28-34$. Eq. riparium Fries Nov. mant. IU. Eq. arvense $\beta$. serotinum Meyer. Chlor. hannov. Eq. campestre Schultz Starg. suppl. 59. Fructificirende Frühlingsstengel, die später grüne Aeste treiben.

Die Stammart auf sandigen und thonigen Aeckern gemein durch d. ganze Gebiet, aus den Niederungen bis in die Bergregion. 24. März und April.

2) Eq. maximum Lamark (1778). Grossscheidiger Schafthalm Garke D. Fl. VI. p. 468. Eq. Telmateja -Ehrh. Hannov. Mag. (1788). "Kannenkraut.“ Koch Syn. p. 964. Rabenhorst Krypt. Eq. ebureum Roth Catal. Eq. fluviatile Schkur t. 168 und mehrer Aut. (nicht Linné). Aendert ab:

Eq. maximum $\beta$. serotinum Al. Braun. In schattigen, feuchten Waldschluchten, an Quellen und Bachufern, zerstreut dch. d. g. Geb. die Var. am Nord - und Ostseestrande, Holland und in Pommern bei Lohme etc. 24. April Mai und später.

3) Eq. silvaticum Lin. Spec. K. Syn. p. 964. WaldSch. Garke D. Flor. Eq. capillare Hoffm. fl. german. Rabenhorst p. 334. Bischoff Krypt. t. 3. fig. 3. Schkur Krypt. t. 166. Feuchte, schattige Waldstellen, Gebüsche, Wiesen, zerstreut, meistens nicht selten, dch. das g. Geb. bis in die Voralpen. Schweiz, Rheingegend bis Belgien, Holland, im Nord- und Mittelgeb. und in ganz Oestr. ausser Dalmat. (nach Vis. Fl. Dalm.).

4) Eq. pratense Ehrh. Beitr. III. (1783 - 88.) (non Roth). Döll. rh. Flor. p. 28. Wiesen-Schafthalm. Rabenhorst Krypt. Milde schles. Krypt. fig. 40-46. Garke D. Fl. p. 468. Eq. umbrosum Mejer (1809) in Willd. Spec. pl. V. Koch. Syn. Eq. sylvaticum $\beta$ minus Wahlb. suec. Schattige, feuchte Stellen an Waldrändern, in Gebüschen, auf Wiesen, Waldäckern mehr im Nord- und Mittelgebiet, aus den Niederungen bis in die Alpenthälern, Piemont, Savoyen, Schweiz, Cant. Wallis im Nicolai - Thal, Graubünden, Rheingegend, selten im Nahethale bei Kreuznach, auf dem Lemberg bei Sobernheim, auf dem Hochwalde bei Stromberg, im Moselthale bei Winningen, Esch bei Eiiskirchen Fl. v. Cöln; Osna- 
briick, Habichsthal bei Kassel, am Unterharze, bei Dessan, Halberstadt, in der Lausitz, Fichtelgebirge, Sachsen Dresden, Brandenburg Berlin, Charlottenburg, Neustadt-Eberswalde, Driesen, Pommern, Mecklenburg häufig, Danzig, Königsberg, Schlesien im Riesengebirge, in den Sudeten und Karpathen, Böhmen, Mähren bei Namiest, Galizien, Oberungarn, Siebenbürgen bei Hermannstadt, Klausenburg, in Salzburg bei Heiligenblut, in der Rauris in Kärnthen, Tyrol bei Innspruck, und Meran (v. Hausm. Tyrol). 24. Mai-Juni.

5) Eq. palustre Lin. Spec. K. Syn. p. 965. SumpfSchafthalm; Garke D. Fl. VI. Döll. rh. Fl. p. 29. Rabenhorst Krypt. Schkr. Krypt. t. 169-170. Bischoff Krypt. t. 3. fig. 1. h. 1. t. 3. Eq. veronense Pollini pl. nov. Veron. III. p. 261. Aendert ab:

b. Eq. palustre C. tenue Döll. rh. Flora. Eq. prostratum Hoppe.

c. Eq. palustre C. membranaceum Fl. Belg. III. Eq. palustre pictum Kickx. In Sümpfen, auf sumpfigen Wiesen, auch auf nassem Sandboden durch das ganze Gebiet gemein. 4. Mai-Juni.

6) Eq. limosum Lin. Spec. K. syn. Schlamm-Sch. Döll. rh. Fl. Garke D. Fl. VI. Rabenh. Krypt. Schkur Krypt. t. 171. Eq. fluviatile Fl. Dan. t. 1184. Lin. suec. secund. Wablb. in Fl. suec. p. 690. Eq. aphyllum Baumg. Trans. IV. Eq. limosum B. minus Al. Br. in Döll. rh. Fl. p. 30 . Eq. uliginosum Mühlenb. ap. Willd. Spec. pl. V. Eq. Heleocharis Ehrh. E. polymorphum Schrank. In sumpfigen Teichen, auf Torfboden, in stehendem und stillfliessendem $W$ asser zerstreut dch. das ganze Geb. bis in die Bergregion. 24. Mai-Juni.

7) Eq. inundatum Lasch in Rabenh. Bot. Centr. Bl. 1846. Eq. arvense- limosum Milde nov. Act. Carl. L. C. 1852. p. 573. fig. $10-20$, 1858. II. Schles. Gesell, 1856. Lasch, Bot. Zeitg. 1857, Oestr. bot. Zeit. 1858. Garke D. Fl. VI. p. 469. Equis. littorale Kühlwein in Rupr. Beitr. IV. Eq. Kochianum Böckl. In Sïmpfen und Sumpfboden stellenweise im Geb. Schweiz am Neuenburger See (Al. Br.) Bremen, Potsdam, Driesen, Breslan, Oderufer bei Auras, Charlottenbrunn, 
Lausitz bei Drebkau etc. Moosbrunn bei Wien, in den Marschauen im Presburger Comitat; am Gesenke bei Nieder - Lindenwiese und am Altfluss in Siebenbürgen. 24.

8) Eq. hiemale Lin. Spec. K. syn. Döll. rh. Fl. Garke D. Fl. VI. Winterschafthalm. Rabenh. Krypt. Schkur Krypt. t. 172 a. z. Theil Eq. hiemale L. Feuchte Wälder, an Ufern, Teichen und sumpfigen Stellen zerstr. dch. d. Gebiet bis in die Gebirge und Alpenländer, Italien, Schweiz, Rheingegend, Baden, Elsass bis Belgien, Holland, Holstein, Mecklenburg bis Preussen, Schlesien im Riesengebirge, im Kessel des Gesenkes, Sachsen, Fichtelgebirge, Würtemberg, Baiern, Oestr. Salzburg, Steiermark, Tyrol und Kroatien; dann in den Karpathen in Galizien, Ungarn, Siebenbïrgen. 24. Juli-August.

9) Eq. trachyodon Al. Braun Flora 1839. K. Syn. p. 967. Rauhzähniger Schafth. Eq. paleaceum Schleich. Catal. 1821, zum Theil nach Bernoulli Schweizer Krypt. p. 76. Eq. hiemale D. trachyodes Döll. rh. Fl. p. 32. Eq. Machaii Newmann. Wohl nur Uebergangsform von Eq. hiemale und Eq. ramosum. Feuchter Sandboden, an Flussufern selten im Gebiet. Bei Constanz im Mollmatinger Ried, in Baden auf der Rheinfläche, Dachslanden bei Carlsruhe, Neu-Breisach, Rheinschanze bei Mannheim nach Speier; Brandenburg bei Berlin, Lettin, Breslau; dann in Tyrol bei Bozen (v. Hausm.). 4 . Juli - August.

10) Eq. ramos um Schleicher Catal. pl. helv. 1807. K. syn. Aestiger Schafth. Schkur Krypt. t. 172 b. Eq. elongatum Willd. Spec. V. Döll. Fl. badens. I. Rabenh. Krypt. Eq. pannonicum W. et Kit. in Willd. Eq. illyricum Hoppe Exsicc. Eq. ramosissimum Desf. atl. 2. (non Humb. et Bonpl.) Eq. procerum Pollin. pl. nov. et $f$. veron. Eq. hiemale C. elongatum Al. Braun Flora 1839. I. Döll. rh. Flora p. 31. Milde Krypt. Schles. Eq. elongatum $\beta$. subverticillatum Al. Braun. Sandboden, Aecker, auch auf feuchten etwas sumpfigen Stellen, zerstr. nicht häufig im südlichen und Mittelgebiet. Italien, Schweiz, Rheinfläche von Basel, Elsass bei Strassburg, Baden bei Alt-Breisach, Rastatt, Ketsch bei Schwetzingen, Mannheim, Offenbach, Pfalz Speier, Maxdorf, Coblenz bei Sebastian- 
Engers und der nordwestlichste Fundort auf der Mühlheimer Heide bei Cöln; dann in Schlesien bei Breslau, Böhmen bei Aussig, Oberöstr. Südsteiermark, Tyrol bei Innspruck, Bozen, Meran, Euganeen, Dalmatien, Venedig, Ungarn und Siebenbürgen. 24. Juli - August.

11) Eq. variegatum Schleich. Catal. pl. helv. 1807. K. Syn. p. 967. Bunter Schafthalm. Döll Flor. badens. I. p. 70. Schkr. Kryptog. tab. 172 b. Eq. tenue Hoppe exsicc. Eq. reptans $\beta$. variegatum Wahlb. lappon. p. 298. Eq. hiemale, E. variegatum Al. Br. Flora 1839, Döll rh. Flora p. 32. Eq. limosum All. Pedem. (non Linné). Etwas feuchte Sandstellen, sehr zerstreut und selten im Gebiet, bis in die Alpenländer, Italien, Schweiz und von Basel durch das Rheinthal, Baden im Breisgau, Kehl vereinzelt bis Mannheim, Worms, Mainz und wieder an der Nordsee in Holland; dann in Hannover am Innerste-Ufer, bei Clausthal in Harze; Schlesien bei Carlowitz und nicht selten Kattern bei Breslau, im Riesengebirge bei Cudowa und am Kessel des Gesenkes, Preussen Wiszniewo bei Löbau; in den Sudeten - und Karpathen - Ländern, in Galizien, Tatra in Ungarn und auf dem Büdös im Szeklerlande, dann in Niederöstr., Salzburg, Steiermark, Kärnthen, Tyrol Vorarlberg, Innspruck, Bozen etc. 24. Juli-August.

12) Eq. alpinum Schkur Oestr. bot. W. Bl. 1857. p. 417. Alpen-Schafthalm. Eq. hiemale alpestre seu Eq. fuseo-zonatum Schkur. Ser. p. 92-93. Auf den Karpathen Siebenbürgens in der Krummholzregion. 24. Sommer.

Anmerkung. Eq. scirpoides, welches Wulfen zufolge einiger sterilen Exemplare im K. K. Hofkabinete in Wien, bei Heiligenblut an d. Möll in Kärnthen fand, hält Neilr. Nachtr. 1861 p. 325, für ein kleines Eq. variegatum. Gleiche Bewandtniss dürfte es nach demselben mit dem Eq. scirpoides haben, welches nach Weber und Mohr in Tyrol vorkommen soll. Das echte Eq. scirpoides Mich. Amer. boreal. II. ist nach Exemplaren von Vermont in America von der Alpenform des Eq. variegatum wenig verschieden. (Welw. Beitr. zur Land - Kunde N. Oestr, IV.). 
B. Hydropterides Wild. Spec. Wasserfarne. Rhizocarpeac Batsch. Marsileaceae Rob. Brow. Koch Syn. p. 967. Schleimfarne.

2. Ordn. Salviniaceen Bartl, ordn. natur. K. Syn. Salvinien.

2) Salvinia Micheli gen. 107. t. 58. K. Syn. p. 968. Salvinie Döll rh. Fl. p. 41.

1) S. natans Hoffm. Deutschl. Flora 1. edit. II. Schwimmende S. K. Syn. Döll rh. Fl. und Fl. bad. I. Garke Deutschl. Fl. VI. p. 470. Sturm II. h. 1. Schkur Krypt. t. 173. Allion etc. Marsilea natans Lin. Stehende und stillfliessende Wasser, Teiche, Seen, zwischen Flossholz, sehr zerstrent im Gebiet. Italien, Piemont im Aosta-Thale, sonst nicht in der Schweiz; auf der Rheinfläche in Baden zwischen Knielingèn und Dachslanden bei Carlsruhe, Schwetzingen im Neckarauer Walde, Mannheim im Hasengraben, Pfalz bei Germersheim, Hochstetten; sonst nicht in den-Rheingegenden, auch nicht in Belgien und Holland, erscheint aber wieder bei Halle, Magdeburg, bei Barby, Brandenburg, Berlin, Potsdam, Schlesien um Breslau, in Weidengebüschen bei Hundsfeld, Ohlau, Oppeln, Frankfurt a/O. Iübeck, Fürstenwalde; Tyrol im Etschthale, Istrien, Ungarn, Galizien, Siebenbürgen, Slavonien und häufig in der Umgebung von Venedig etc. 24. Juni-August.

3. Ordn. Marsileaceen Bartl. ordn. natur. K. Syn. p. 967. Döll rh. Fl. p. 42 .

3) Pilularia Lin. gen. K. Syn. Döll rh. Flora p. 43. Pillenkraut.

1) P. globulifera Lin. Spec. K. Syn. p. 968. Döll rh. Fl. Kugelfrüchtiges P. Garke, Deutschl. Fl. VI. p. 470. Sturm II. h. 1. Schkur Krypt. t. 173. In stehenden und stillfliessenden Gewässern, Teichen, Sümpfen, Wiesengräben sehr zerstreut durch das Gebiet; verbreitet in Nordwest-Deutschland. Italien, Schweiz, Bonfol bei Pruntrut, auf der Rheinfläche, Oberelsass bei Colmar, Stras̄sburg, Baden bei Rastatt, Carlsruhe, Pfalz, Speier, Kaiserslautern, Frankfurt, Hanan, Nassau, am Seeburger Weiher, fehlt dann am Mittelrhein bis Siegburg, bei Bonn und Merrheim bei Cöln, findet sich dann dch. die 
niederrheinische Ebene bis Belgien bei Antwerpen und Holland nach Westphalen durch das nordwestliche Deutschland; dann in Franken bei Erlangen, in der Lausitz, in Böhmen, Mähren, Ungarn jenseits der Theiss und in Siebenbürgen. 4. August - September.

4) Marsilea Lin. gen. pl. K. Syn. p. 968. Marsilie.

1) M. quadrifolia Lin. Spec. Koch Syn. Vierblätterige M. Döll rh. Flora p. 44. Fl. badens. I. p. 87. Sturm II. h. 1. Schkur Krypt. t. 173. Bischoff Krypt. t. 7-8. Stehende und langsam fliessende Wasser, an schlammigen Ufern und in Gräben. Italien, Schweiz bei Villeneuve und Bonfol bei Pruntrut, Rheinthal, im Elsass Ischenheim bei Strassburg bis Germersheim in der Pfalz; Baden bei Kork, Kehl, Rastatt, Dachsland, Neckerauer - Wald bei Mannheim, Speier, sonst nicht am Rheine, auch nicht in Belgien und Holland und fehlt auch in Norddeutschland. Dann findet sich die Pflanze wieder in Oberbaiern zwischen Rosenheim und Kloster Roth, Oberöstr. im Hausrückkreise, Salzburg im Klagenfurter See, Steiermark, Tyrol am Gardasee, Krain, Ungarn im Comit. Zala, Baranya, Essek in Slavonien, Galizien, Siebenbürgen und um Venedig. 24. Juli-September.

C. Bryopterides Alex. Braun. Döll rh. Flora p. 34. Selagines Endl. Syst. Moosfarne.

4. Ordn. Lycopodiaceen D. C. Fl. Fr. 2. Bärlappgewächse. Koch Syn. p. 969. Döll rh. Fl p. 34.

a) Is o eteen Bartl. ordn. natur. Garke Deutschl. Flor. p. 470. Koch Syn. p. 969. Döll rh. Fl. p. 39. Brachsenkräuter.

5) Is oetes Lin. gen. Koch Syn. Brachsenkraut.

1) I. lacustris L. Spec. K. Syn. Döll rh. Fl. Sumpfbrachsenkrant. Garke D. Fl. edit. VI. Rabenhorst Krypt. Rchb. Deutschl. Flora. Icones Spitzkeimer. I. Band. p. 1. fig. 1. Sturm II. h. 17. Schkr. Krypt. t. 173. Tausch Flora 1819. II. In etwas hochgelegenen Seen, Teichen, unter dem Wasser auf dem Grunde wachsend, selten im Gebiete. Italien im langen See, auf dem Schwarzwalde in Oberbaden im Feld und Titi-See bei Freiburg; Elsass in den Vogesen-Seen bei 
Gerardmer, Longemer und im schwarzen See; Holland im Udelermer, Schleswig im Falkwater-See, Holstein im Einfelder-See bei Kiel, in den Seen bei Trittau unweit Hamburg, Plöschen See bei Ratzeburg, Hannover im See bei Celle, auf Usedom in den Krebs - Seen, bei Heringsdorf im See, bei Bülow in Pommern, Landseen in Westpreussen bei Danzig; Böhmen im Bistritzer und Eisensteiner-See im Böhmerwalde, um Krakau im See $\mathrm{Sz}$-Ivany und Dobaka in Siebenbürgen. 24. Juli-September.

2) I. echinospora Durien in Greml. Fl. excur. d. Schweiz 1867. Blätter feiner zugespitzt, Makrosporen dicht mit feinen, stacheligen Fortsätzen versehen. Piemont in Lago maggiore entdeckt, anch soll die Form oder Art im Breisgau in Feldsee gefunden sein. 4. Sommer.

b) Lycopodineen Bartl. ordn. natur. Landpflanzen von der Tracht der Laubmoose. Döll rh. Flora p. 35.

6) L y copodium Lin. gen. Koch Syn. p. 969. Springer. Bärlapp.

1) L. Selag o Lin. Spec. K. Syn. Garke D. Fl. VI. p. 471. Tannen-Bärlapp. Rabenhorst Krypt. Sturm II. h. 5. Schkur Krypt. t. 159. L. recurvum Kit. in Willd. Sp. pl. V. L. reflexum Schkr. An etwas feuchten, mit Moos bewachsenen Stellen, Schluchten und Felsen der Hochgebirge bis in die Alpenregion, seltener in Niederungen, zerstrent dch. das Gebiet. Italien, Savoyen, Schweiz, Elsass in den Vogesen, Baden auf d. Schwarzwalde, Nassau, Nahethal, Hochwald, Trier, Eifel, Hohes Veen, Ardennen in Belgien; Siegburg bei Bonn, Holland, Bremen, Westphalen, Hessen, Harz, Franken, Schlesien und ausser Dalmatien stellenw. durch ganz Oestr. 24. JuliAugust.

2) L. inundatum Lin. Sp. K. syn. p. 970. Döll th. Fl. p. 38. Sumpf-Bärlapp. Garke D. Fl. VI. Rabenh. Krypt. Sturm II, h. 5. Schkr. Krypt. t. 160. Torfsümpfe, in Torfstichen, feuchte Sandstellen in Heidegegenden und auf Hochmooren, zerstreut durch das Geb. Schweiz, Elsass, Baden, Pfalz, Trier, Luxemburg bis Belgien, Nassau und stellenw. durch die Rheingegend bis in die Niederlande, Westphalen, Han- 
nover, Bremen, Lübeck etc. nach Norddeutschland; dann in der Lausitz, im Erzgebirge, Sachsen, Fichtelgebirge, Baiern, Würtemberg; Böhmerwald, Oestr. Salzburg, Tyrol bis Verona etc. 24. Juli-August.

3) L. a nnotinum Lin. Sp. Koch Syn. Sprossender B. Döll rh. Fl. p. 38. Garke D. Fl. VI. Rabenhorst Krypt. Schkr. Krypt. t. 162. Sturm II. h. 5. Bischoff Krypt. Gew. t. 16. W.älder, Heiden des Hochgebirges bis in die Voralpen und besonders in den nördlichen Ländern in die Niederungen herabsteigend; zerstr. dch. das Gebiet nicht selten. 24. Juli-August.

4) L. alpinum Lin. Spec. Koch Syn. p. 970. Alpen Bärlapp. Garke D. Fl. VI. Rabenhorst Krypt. Schkr. Krypt. t. 161. Sturm II h. 5. Nach Springer Flora 1839. I. p. 179, Bernoulli Krypt. d. Schweiz 1847, soll L. alpinum L. Var. von L. complanatum sein? Begrasste Stellen der Hochgebirgskämme und der Alpen. Piemont, Savoyen, Schweiz, Tyrol, Salzburg, Ober - und Niederöstr., Steiermark, Kärnthen. Dann auf den Vogesen im Elsass am Hoheneck, am Seebuck; Baden auf den höchsten Kämmen des Schwarzwaldes, auf dem Feldberg; Westphalen auf dem Astenberg, Harz, Brocken, Erzgebirge bei Gottesgabe, Schlesien im Riesengebirge an der Schneekuppe, am hohen Rade im Mährischen Gesenke, am Altvater, auf den Sudeten und Karpathen in Ungarn, Galizien und Siebenbürgen. 24. August-September.

5) L. complanatum L. Sp. K. Syn. p. 971. Flachblätteriger Bärlapp. Garke D. Fl. VI. p. 472. Rabenh. Krypt. Sturm II. h. 5. Schkur Krypt. t. 163; Milde schles. Krypt. p. 404. fig. 7 - 8. Aeussere Stengelbl. lanzettlich, immer pfriemlich, kleiner.

6) L. Chamaecyparissus Alex. Braun in Döll rh. Fl. p. 36, als Art Koch Syn. Rabenhorst, Milde. Stengelblättchen gleichgestaltig. $L$. complanatum $\beta$ Chamaecyparissus Döll Fl. badens. I. p. 80, v. Hausmann Tyrol, Springer Lycopod. II. p. 47. Ruprecht Beitr. III. Milde Oestr. bot. Zeit. 1859. Garke D. Fl. VI. p. 472, ist von Lycopodium complanatum Lin. Pollich und der meisten Floristen nicht als Art verschieden. Wälder, Heiden der Berg - und Voralpen-Gegenden und im 
nördlichen Gebiete auf Sandboden bis in dic Niederungen, zerstr. dch. das Geb. Ausser Dalmatien auch in allen Ländern Oestr. 24. Juli-August.

6) L. clavatum Lin. Spec. Koch Syn. p. 971. Keulenförmiger Bärl. Döll. rh. Flora, Garke D. Fl. VI., Rabenhorst Kryptogam. p. 330. Sturm II. h. 5. Schkr. Krypt. t. 162. Waldige Stellen, Heiden, Bergabhängen bis in die Alpen, zerstreut dch. das ganze Geb. 24. Juli--August.

7) Selaginella Springer Döll rh. Fl. p. 38. Selaginell, Bärläppchen.

1) S. spinulosa Al. Braun K. Syn. p. 971. Döll. rh. Fl. Garke D. Fl. VI. p. 472 . Wimperzähnige S. Selaginella selaginoides Link Filices p. 158. Rabenh. Krypt. L y copodium selaginoides Lin. Sp. Sturm II. h. 5. Schkur Krypt. t. 165. Bischoff Krypt. t. 11. An etwas feuchten, mit Gras oder mit Moos bewachsenen Stellen bis in die Alpenregion, seltener auf Moorboden, wie in Oberbaden unter Torfmoos am Ufer des Feldsees, in Sumpfe bei dem Seebaur im Breisgau und sonst noch auf den höchsten Punkten d. Schwarzwaldes. Auf den Alpen in Piemont, Schweiz und durch die Alpenkette nach Tyrol, Oestr. Baiern auch auf der Heide bei Ismaning, Isarauen bei München; an Quellen am Wege von Rattersdorf nach dem Zeitzgrunde bei Jena, auf dem Harze, dem Brocken, im Erzgebirge am Fichtelberge; Schlesien im Riesengebirge beim Rübenzahlgarten und Aupengrund, im Mährischen Gesenke am Klosterberge, Petersteine und im grossen Kessel; Wien auf dem Schneeberge; dann in den Karpathen und an noch vielen Stellen in Oestr., fehlt aber in Dalmatien und Kroatien. 24. Juli-August.

2) S. helvetica Link. Filices 1841. Springer in Döll rh. Fl. 1843. Schweizer Selaginelle. K. Syn. p. 971. Rabenhorst Krypt. p. 330. Ly copodium helveticum Lin. Spec. Jacq. Austr. t. 196. Schkur Krypt. t. 165. Lycop. radicans Schrank. Felsen, Wege, Triften der Voralpengegenden, bis zum Fusse der Alpen herabsteigend, durch die Alpenkette. Italien, Piemont, Savoyen, Schweiz, auch im Cant. St. Gallen am Rheindamme bei Rheineck, auf den hohen Vo- 
gesen, München in den Auen und Triften; um Salzburg, in Böhmen, Wien bei Dornbach und im Prater, Oestr. in fast allen Alpenländer, in Siebenbürgen und in Kroatien, Venetien; aber nicht in Dalmatien. 24. Juli - August.

3) S. denticulata Link Filices 1841. Gezähnte Selaginelle. Springer Lycopodiaceen 1842. II. p. 82. Rabenh. Krypt. p. 331. L y copodium denticulatum Lin. Spec. Dill. Hist. muscor. t. 66. fig. 1.a. Diese eigentliche Mittelmeer-Pflanze, findet sich an schattigen, steinigen Stellen durch ganz Dalmatien und ist die einzige Lycopodiacee, welche in diesem Lande vorkommt; ob auch nach Baumg. Trans, und Schkur Oestr. bot. Zeitg. auf den hohen Karpathen von Rodna in Siebenbürgen dürfte nach Neilr. Beitr. p. 339. unrichtig sein. 4. Sommer.

D. Fi lices Lin. genera pl. edit. 4, exclusiv. generibus Rob. Brown prodr. p. 146. Filices frond osae Laubfarne. 1. Ordn. Ophioglossaceen Rob. Brow. prod. p. 162. Natterzungengewächse Koch Syn, p. 972. Stachyopterides Willd. Aehrenfarne.

8) Botrychium Swartz Syn. Filicum. Koch Syn. Döll rh. Flora. Mondraute.

1) B. Lunaria Sw. Filic. p. 110, K. Syn. Gemeine Mondraute. Döll rh. Fl. p. 24. Garke D. Fl. VI. p. 472. Rabenhorst Krypt. 326. Schkur Krypt. t. 154. Milde schles. Krypt. p. 657, fig. 124-37. Osmunda Lunaria Lin. Spec. Fl. Dan. Sturm II. h. 1. Strempel Filic. Berol. fig. 1 -4. Heiden, grasige Abhänge bis in die Voralpen zerstr. dch. d. g. Gebiet. 24. Mai-Juni.

2) B. simplex Hitchcok in Sillim. Americ. Journal 1823. nach Milde Krypt. Schles. p. 164, fig. 138-74. Röper Bot. Zeitg. 1859. B. Kannenbergii Klinsm. Bot. Zeit. 1852, p. 379, t. 6. Lasch Bot. Zeit. 1856. Garke D. Fl. VI. p. 473. Einfache Mondraute. Vielleicht nur Var, von voriger, B. Lunaria $\beta$. cordatum Fries Nov. Mant. II. p. 63. Sandige Stellen zwischen Gebüschen am Ufer der Swiage bei Memol, auf dem Anger bei Driesen, bei Neudamm, selten bei Rostock und in Mährischen Gesenlie bei Niederlindenwiese. 24. Juni. 
3) B. matricariaefolium Al. Braun in Döll rb. Flora p. 24. Koch Syn. p. 972. Kamillenblätterige Mondraute. Milde schles. Krypt. p. 659. fig. 182-96. Röper Bot. Zeit. 1859. B. rutaceum Willd. Spec. pl. V. Garke D. Fl. VI. Schkr. t. 155, fig. 6, nicht Wahlenb. Swartz Filic. p. 110, (exclusive Var. multifido folio). Osmunda Iunaria Fl. Dan. t. 18. fig. rechts. Var. des B. Lunaria $\beta$ rutaceum Wahlb. suec. II. B. Lunaria D. rutaefolium. Fries Summ. veget. Röper Mecklenb. I. p. 111. Rabenh. Krypt. Döll Fl. badensis I. p. 52. B. Reuteri Payot nach Milde. Trockene, sonnige Stellen, auf Triften, Wiesen in Niederungen und Vorgebirgen sehr zerstr. selten und meistens einzeln durch das $\mathrm{Ge}_{e}$ biet. Schweiz im Veltlin bei den Bädern von Bormio, im Chamouny-Thale und auf d. Bernhardin; Elsass in den Vogesen am Hoheneck bei Ribeauviller, Bruyéres und bei Bitsch; Baden zwischen Schwetzingen und Mannheim, in Franken bei Erlangen, Sachsen; Schlesien im Gesenke bei Nieder-Lindenwiese, Böhmen im Prager Kreise; bei Graz; Ungarn auf der Jaworina im Comit. Neutra; im Mährischen Gesenke, in Galizien und in Siebenbürgen. 24. Mai-Juni.

4) B. rutaefolium Al. Braun Döll rh. Flora p. 24. Koch Syn. 972. Rautenblätterige Mondraute. Milde schles. Krypt. p. 590, fig. $197-200$. Döll Flora baden. I. Röper Bot. Zeitg. 1859. B. rutaceum var. multifido folio Swartz Filic. p. 111. B. matricarioides Willd. Spec. pl. V. Rabenhorst Krypt. p. 327. B. rutaceum Wahlenb. suec. II. B. Matricaria Sprengl. IV. p. 22. Garke D. Flora VI. p. 473. Osmunda Matricariae Schrank Baier. Flora II. Sturm II. h. 6. Osmunda Lunaria Flor. Dan. t. 18, die mittlere Figur.

Mit Gras bewachsene Waldstellen, Berglehnen, Heiden, Wiesen, etwas feuchten Sandboden, selten und nur stellenweise im Gebiete. Schweiz im Chamouny-Thale (Gremli Flor. excurs. der Schweiz 1867). Elsass auf den Vogesen bei der Belchenhütte des Ballons von Sultz, im Munsterthale am rothen Rücken hinter Sanderach. Berlin am Gesundbrunnen, Driesen, Mecklenburg bei Dragun, Warnemünde an den Dünen der Ostsee, Dierhagen und Neuhans; Stettin, Thorn, 
Danzig, Memel; Schlesien bei Garsuche, bei Ohlau, Gnadenfeld; im Mährischen Gesenke am Uhustein, bei Einsiedel an der Lissa Hora; Böhmen bei Woleschna; Lausitz bei Nisky, bei Reichenbach im Bunzlauer Kreise und im Iglauer Kreise; Grenz-Karpathen, Ungarn im Comitat Zips und in Siebenbürgen; auf den Alpen in Salzburg, in der Satnitz bei Klagenfurt und auf dem Bacher-Gebirge in Tyrol. 24. Juni-Juli.

5) B. virginianum SW. Filic. p. 111. Virginische Mondraute. B. anthemoides Presl Böhm. Gesell.der Wissensenschaft. 1847. Zool. bot. Ver. 1857. Milde schles. Krypt. p. 699. fig. 201. Oestr. bot. Zeit. 1858. Gremli Fl. exc. d. Schweiz 1867. Auf Waldwiesen sehr selten im Gebiet. Schweiz im Cant. Graubünden, im Prättigau gegenüber dem Serneuser Bade. Früher auch von Presl in Oberöstreich auf dem Pürn (Pyrrhn) bei dem Spital nach Milde entdeckt, doch später nicht mehr und scheint dort verschwunden. 4. Juni-Juli.

9) Ophioglossu m Linn. Gen. Natterzunge. Koch Syn. p. 973. Döll rh. Flora 1823.

1) Oph. vulgatum Linn. Gemeine Natterzunge, Koch Syn. Döll rh. Fl. Garke D. Fl. p. 473. Rabenhorst Krypt. Sturm II. h. 6. Schkur Krypt t. 153. Wiesen und mit Gras bewachsene Heiden, aus den Niederungen bis in die Gebirge, zerstreut durch das ganze Gebiet. 4. Juni-Juli.

2) Oph. lusitanicu m Linn. Portugalische Natterzunge. Koch Syn. p. 973. Lamark Illustrat. IV. A. 864. fig. 3. Barrel t. 252. Lowe Ferns VII. A. 45. Sandige Stellen in Istrien am Hafen von Veruda auf dem Scoglio St. Clementi bei Lesina in Dalmatien. 24. Juni.

2. Ordn. Osmundaceen Rob. Brown' Prodr. Rispenfarne. Koch Syn. p. 973. Schizopterides Willd. Sp. pl. V. Döll rh. Flor. p. 22.

10) Osmunda Lin. gen. Koch Syn. Döll rh. Flor. Traubenfarn.

1) 0. regalis Lin. Spec. Königsfarn. Koch Syn. Döll rh. Flor. Garke D. L. Flora p. 473. Rabenhorst Krypt. p. 325. Sturm II. h. 6. Schkur Krypt. t. 145. Torfboden auf Wald- 
wiesen, Heiden der Niederungen und Berggegenden, zerstreut im Gebiet, aber in vielen Ländern fehlend.

Italien, Schweiz, Würtemberg; Oberbaden bei Freiburg im Breisgau; Elsass auf dem Hochfelde, Hagenauer Forst, bei Bitsch, Pfalzburg; bei Zweibriicken und Kaiserslautern, in der Saar-Gegend; Luxemburg stellenw. bis Neuerburg in der Eifel; dann wieder in der niederrheinischen Ebene bei Siegburg und Mühlheim bei Cöln und von dort zerstr. bis Belgien und Holland; Westphalen, Oldenburg, Bremen, Mecklenburg, Halle, Halberstadt; Sachsen bei Dresden, Schlesien etc. Oestreich in der Prein bei Wien, am Schneeberge; Südtyrol bei Valsugana, bei Bassano, auf den Lessenischen Bergen, in den Euganeen im Venet.; dann im Szeklerlande an der Hargita, am Büdos und bei Krakan etc. 24. Juni-Juli.

3. Ordn. Hymenophyllaceen Endl. Syst. Hautfarne. Garke D. L. Flor. Uebersicht der Familien p. 108.

11) Hymenophyll n m Smith Engl. Bot. Hautfarn.

1) H. tunbridgense Sm. E. Bot. t. 162. (1794). Tunbridger Hautfarn. Garke D. Fl. VI. p. 474. Rabenhorst Krypt. p. 309. Schkur Krypt. t. 135 d. Trichomanes tunbridgense Lin. Spec. Felsen, Schluchten in der Sächsischen Schweiz an einer Stelle des Uttewalder Grundes am Teufelthor; dann im nördlichen Friaul und bei Artegna in der Praetur Gemona in Carnia, nicht in Kärnthen. 24. August.

4. Ordn. Polypodiaceen Rob. Brow. Prodr. p. 145. Koch Syn. p. 974. Tüpfelfarne.

12) Grammitis Swartz Syn. Filic. Syn. Vollfarn.

1) G. Ceterach Sw. K. Syn. p. 974. Schuppen-Vollfarn. Garke D. Fl. VI. p. 474. Döll rh. Flor. p. 3. Asplenium Ceterach Lin. Sturm II. h. 5. Scolopendrium Ceterach Engl. Bot. Roth tent. 3. p. 48. Ceterach officinarum Willd. Berol. II. p. 1068. Felsen, Mauern in niederen und gebirgigen Gegenden vorzugsweise im südlichen Gebiete und im Weststriche. Italien, Savoyen, Schweiz; Rheingegenden an vielen Stellen in Elsass, Baden, in d. Pfalz, in Nassau, an der Nahe, Mosel, Trier, Luxemburg, Coblenz bis zum Siebengebirge und noch an der Neander-Höhle bei Diisseldnrf selten, an der 
Maas in Belgien; dann in Tyrol, Vorarlberg bis zum Baldo, um Görz; Istrien, Südkrain, Venedig, Kroatien, Dalmatien, Ungarn, Siebenbürgen etc. 24. Juni-Juli.

13) Gymnogramme Desveaux Berl. Magac. V. Nachtfarn.

1) G. le p to phylla Desv. B. M. Schmalwedeliger Nacktfarn. Rabenhorst Krypt. p. 311. v. Hausmann Tyrol 1512. Polypodium leptophyllum Linn. Spec. Schkur Krypt. t. 26. Grammitis leptophylla Sw. Syn. Filic. Acrostichum leptophyllum De Cand. Felsen, Mauern im südlichen Gebiete. Italien, Südtyrol bei Meran und im Val Daone in der Giudicarie, dann in Dalmatien um Ragusa. 4. März-April:

14) Polyp odium Linn. Gen. Koch Syn. p. 974. Döll rh. Flora p. 4. Tüpfelfarn.

1) P. vulga re Linn. Spec. Koch Syn. Döll rb. Flora. Gemeiner Tüpfelfarn (Engelsüsswurzel). Garke D. Fl. VI. p. 474. Rabenh. Krypt. p. 310. Sturm II. h. 1. Schkur Krypt. t. 11. Felsen, Waldschluchten auf Baumwurzeln, Manern zerstreut durch das ganze Gebiet. 24. Juni-Juli.

2) P. Phegopteris Linn. Spec. Koch Syn. Döll rh. Flor. p. 5. Buchen-Tüpfelfarn. Rabenhorst Krypt. Schkur Krypt. t. 20. Laubwälder, Gebüschen, besonders in Buchenwaldungen zerstreut durch das ganze Gebiet bis in die Voralpen; scheint in den südlichen Küstenländern zu fehlen. $\mathrm{Phe}$ gopteris polypodioides Fée Garke D. Flor. VI. p. 474. 4. Juli-August.

3) P. Dry opteris Linn. Spec. Koch Syn. Döll rh. Fl. p. 6. Eichen - Tüpfelfarn. Rabenh. Krypt. p. 311. Sturm II. h. 1. Schkur Krypt. t. 25. Phegopteris Dryopteris Fée Garke D. Flor. VI. Gren. et Godr. Fl. Franc. III. Bert Krypt. p. 46. Var. Polyp. Dryopteris v. disjunctum Ruprecht Polyp. disjunctum Schkur Oestr. bot. Zeit. 1858. Schattige Laubwälder, besonders in Eichenwaldungen zerstrent dch. d. g. Gebiet. Var. in Siebenbürgen bei Kronstadt. (Schieferform). 4. Juli-August.

4) P. robertianum Hoffm. D. Fl. I. (1795). Kalkliebender Tüpfelfarn. Koch Syn. p. 974. Polypodium calcareum Sm. 
Brit. III. Döll rh. Fl. p. 6. Rabenhorst Krypt. p. 310. Polypod. Dryopteris Bolt. Filic. p. 53. tab. 1. Polypod. Dryopteris $\beta$. glandulosum Neilr. Phegopteris robertianum Alex. Braun. Garke D. Fl. VI. p. 475. Steinige Gebirgswiilder, besonders auf Kalkgestein an Felsen, in Schluchten, Mauern zerstreut durch das Gebiet bis in die Voralpen. Italien, Savoyen, Schweiz mit dem Jura-Zuge nach Elsass, Würtemberg, Baden anf dem Kastelberge bei Sulzburg im Breisgau, Karlsruhe, Durlach, Schwetzingen nach Rheinhessen, Nassau bei Weilburg und von St. Goar stellenw. bis Tönnestein, bis Cöln; Obermosel Trier, Schengen Luxemburg bis Belgien, Verviers Dolhain, Westphalen, Harz bei Rübeland, Magdeburg, Dessau, Potsdam, Sachsen, Schlesien, Baiern an d. Donau und Isar; Oestr. am Schneeberg bei Wien, Tyrol und zerstr. durch alle Länder Oestr. 24. Juli-August.

15) Woodsia Rob. Brow. Transact. of the Linn. societ. 1816. Woodsie.

1) W. ilvensis, R. Br. Garke D. Fl. VI, p. 475. Südliche W. Rabenhorst Krypt. p. 320. Milde Schles. Krypt. p. 618. Lowe Ferns VII. tab. 28. W. hyperborea $\beta$ rufidula Koch Syn. p. 975. Acrostichum ilvense Lin. Acrost. Marantae, Hanke in Jacq. Coll. II. nicht Linné. Polypodium ilvense Sw. Filic. p. 27. Sturm II. h. 6. Schkur Krypt. t. 19. Polyp. Marantae Hoffm. D. Fl. II. Polystichum Marantae Roth tent. 3. Aspidium rufidulum SW. Nephrodium rufidulum Mich. Amer. 2. Felsige Orte, Gebirgsabhänge bis in dic Alpenregion, nur stellenweise und selten im Gebiet. Alpen der Schweiz, Nordtyrol, Salzburg, Steiermark, Ungarn in der Zips, bei Hont, auf dem Fogaras in Siebenbürgen, in Kroatien und Dalmatien, Slavonien bei Velika; Rhön-Gebirge auf der Milseburg, Burghaltungen bei Kassel, Harz im Ocker - und Bode-Thale; Oberlausitz am Schlosse Tollenstein; Böhmen Tissamühle bei Einsiedel unweit Karlsbad, am Milleschauer bei Töplitz, Kaurzimeg an der Bernau, an dem Hirschberge bei Niemes, Iglau auf dem Hasensprung, Schlesien im Weistritz-'Thale. 24. Juli-August. 
2) W. hyperborea Rob. Brow. Prodr. p. 173 t. 2. Garke D. Fl. VI. p. 475. Nördliche Woodsie. W. hyperborea a Koch Syn. p. 975. Rabenhorst p. 320. Milde Schles. Krypt. p. 621. Polypodium aronicum Wither. Smith Brit. 3. Polypod. hyperboreum Liljebl. Ceterach alpinum De Cand. Fl. Franc. Felsen der Alpen, Voralpen und im Riesengebirge in Schlesien an den Basaltfelsen der kleinen Schneegrube und im Kessel des Gesenkes selten. Alpen der Schweiz, in Tyrol im Pusterthale, auf dem Schlern, bei Meran etc. Salzburg, Kärnthen. 24. Juli-Agust.

3) W. glabella Rob. Brow. in Richards app. of Frankl. Journal 1823. Geglättete Woodsie. Milde Krypt. Schles. p. 624. fig. 104. W. pulchella Bertol. Krypt. p. 111., die amerikanische Pflanze soll von der Tyroler verschieden sein. Neilr. Nachtr. p. 127. Südtyrol auf der Alpe Kreuzberg und auf den Dolomitfelsen am alpinen Praxer-See im Pusterthale. 4. Juli-August.

16) Aspidium Swartz Syn. Filic. Rob. Brow. Prodr. Flor. nov. Holland. edit. Norimb. Koch Syn. p. 976. Döll rh. Fl. p. 15. Schildfarn.

1) A. Longitis Sw. Filic. p. 30. K. Syn. Döll rh. Fl. p. 19. Garke D. Fl. VI. p. 476. Scharfer Schildfarn. Rabenhorst Krypt. p. 323. Schkur Krypt. t. 29. Polypodium Longitis Linn. Spec. Sturm II. h. 1. Polystichum Longitis Roth tent. 3. Bewaldete Felsen der Alpen bis in die Krummholzregion, zerstreut durch die Hochgebirge und die Voralpenketten. Piemont, Savoyen, Schweiz, Vorarlberg, Tyrol bis Valsugana, Salzburg etc. Im Schweizer und Badener-Jura, am Wasserfall bei Basel, auf der rauhen Alp, auf dem Feldberge; Elsass auf den hohen Vogesen, dem Rossberge und bei Reichenstein; Vogelsberg in der Wetterau im Fichtelgebirge; Schlesien im Riesengebirge, Riesengrund, Kiesberge, im grossen Kessel des Mährischen Gesenkes; im Harz; Sachsen im Uttewalder Grunde; Baiern bei Pullach etc. Würtemberg; Oestr. im Höllenthale bei Wien und stellenw. in allen Ländern Oestreichs. 24. Juli-August. 
Anmerkung. Die Angaben von Gent und Hübener in Nassau und bei Altenahr sind sehr zweifelhaft, da die Pflanze später nicht mehr gefunden worden ist.

2) A. Lobatum Sw. Syn. Filic. Kunze Flora 1848. I. Garke D. Fl. VI. Gelappter Schildfarn. Sm. Engl. Bot. t. 1563. Schkur Krypt. t. 40. Milde schles. Krypt. p. 490. fig. 81, 87 - 93, 97. Polystichum lobatum Presl. As. aculeatum a) vulgare Döll rh. Flora p. 20. Koch Syn. p. 976. Asp. aculeatum Sturm II. h. 11. Polypodium Plukenetii Loisl. not. 146 nach Gren. et Godr. Fl. Fr. III. Wälder, Bergschluchten, Abhänge zerstreut durch den gebirgigen Theil des Gebietes bis in die Voralpenregion. Italien, Schweiz, Rheingebirge, Elsass, Baden, Nassan, Coblenz, Bonn, Cöln, Trier, Luxemburg bis Mastrich am Petersberg, Belgien, Westphalen, Hannover, Harz, Jena, Sachsen, Landskrone bei Görlitz, Mecklenburg, Insel Rügen bei Ralswick, Brandenburg, Niederlausitz; Schlesien bei Breslau und häufig im Riesengebirge; in den Sudeten und Karpathen; Würtemberg, Baiern bei Erlangen und im Isar Thale; Oestr. zerstreut durch alle Provinzen. 24. Juli - August.

3) A. a culeatum Sw. Syn. Filic. p. 37. Kunze Flora 1848. I. Garke D. Flora VI. Stacheliger Schildfarn. Sm. Engl. Bot. t. 1562. Willd. Spec. 5. Rabenhorst Krypt. Milde schles. Krypt. p. 501. fig. 82-83. Polypodium aculeatum Lin. Spec. Polystichum -- Roth, Presl. Aspidinm aculeatum $\beta$. Swarzianum Koch Syn. p. 976. Waldschluchten, Berglehnen, Felsen selten. Italien, Schweiz, Oberbaden, Belgien bei Spaa, Schlesien im Gesenke bei Ustron und im südlichen Siebenbürgen; wohl noch weiter verbreitet. 24. Juli-August.

4) A. angulare Kit. in Willd. Spec. pl. V. Garke D. Fl. VI. p. 477. Zackiger Schildfarn. A. Braunii Spenn. Fl. Frib. Kunze, Rabenhorst Krypt. Milde Schlesiens Krypt. fig. 84-86, 94-95. Polystichum angulare Presl. pter. Asp. aculeatum Schultz Fl. gall. et german. exsicc. Nr. 571. Asp. aculeatum b) angulare Döll Asp. aculeatum c) Braunii Döll rh. Fl. p. 21. Koch Syn. p. 977. Aspidium pilosum Schur Siebenb. Verh. 1851 - 59. Oestr. but. Zeit. 1858. Bergwälder der Vor- und Hochgebirge, selten im Gebiet. Schweiz im 
Haslithale an Felsen des Engelsberges und weiter verbreitet; Oberbaden an Porphyrfelsen am Iberge bei Baden, Belgien bci Spaa; Sächsische Schweiz im Wehlener und UttewalderGrunde; Schlesien an Waldlehnen bei Ustron verbreitet, seltener am Hockschar im Kessel des Mährischen Gesenkes und am Schlossberge bei Zuckmantel, auf den Sudeten und Karpathen in Ungarn, Marmoros, Siebenbürgen und im Alpenzuge von Salzburg, in Steiermark im Bachergebirge, Kroatien, Dalmatien und wohl weiter verbreitet. 24. Juli-Angust.

NB. Döll, Koch u. Milde vereinigen die obigen 3 Arten unter Aspidium aculeatum Swartz.

17) Polyatichum Roth tent. Flor. germ. 3. Koch Syn. p. 977. Punktfarn.

1) P. Thelypteris Roth tent. Koch Syn. Garke D. Fl. VI. Sumpf-Punktfarn, Aspidium Thelypteris Sw. Filic. Rabenhorst Krypt. Schkur Krypt. t. 52. Döll rh. Fl. p. 19. Polypodium Thelypt. Lin. Mant. Sturm II. h. 1. Acrostichum Thelypt. Lin. Spec. pl. Lastrea Thelypt. Presl. Sumpfige, torfige Waldwiesen, Erlengebïsche in den Ebenen und Berggegenden, zerstreut durch das ganze Gebiet. 4. Juli - August.

2) P. Ore opter is D. Cand. Fl. Fr. 2. Koch Syn. p. 978. Berg-Punktfarn. Garke D. Fl. VI. p. 477. Polypodium Oreopteris Ehrh., Polypodium motanum Vogler dissert. Gies. 1781. Polyp. limbospermum Allion auct. p. 49. Polyp. pteriodes Vill. Delph. 4. Aspidium Oreopteris Sw. Syn. Filic. Döll rh. Fl. Rabenhorst Krypt. Schkur Krypt. t. 52. Aspid. montanum Acherson. Lastrea Oreopteris Presl. In trocknen oder auch etwas feuchten, torfhaltigen Wäldern, besonders in Nadelholzwälder der Berggegenden bis in die Voralpen, zerstreut durch das Gebiet, mit Ausschluss der südlichen Küstenländer. 24. Juli - August.

3) P. Filix mas Roth tent. III. Koch Syn. p. 978. Wurm-Punktfarn. Aspidium Filix mas Sw. Syn. Filic. Rabenhorst p. 321. Schkur Kryptog. t. 44. Asp. cristatum Pollin. Ver. III. (nicht Swartz) Asp. Mildeanum Göppert. Polypodium Filix mas Lin. Spec. (Polystichum affine Ledebur Ross. IV. Aspidium affine Fischer et Meyer und Ruprecht Beiträge, 
welche als eine bekannte Pflanze bei Cattaro in Dalmatien vorkommt) hält Neilr. Beiträge p. 329 für eine Var. der Hauptart. Aspidium Filix mas B. erosum Döll rh. Fl. p. 16. Asp. depastum und Asp. erosum Schkur Krypt. t. 45-51. Polypodium Heleopteris Borkh. in Römers Archiv sind nach Koch aus der Art geschlagene monströse Formen. Steinige Wälder, Gebüsche, Schluchten, Hohlwege durch das ganze Gebiet, nicht selten. 24. Juli-August.

4) P. cristatum Roth tent. Fl. germ. III. Koch Syn. Kammartiger Punktfarn. Garke D. Fl. VI. p. 478. Aspidium cristatum Sw. Syn. Filic. p. 52. Döll rh. Fl. p. 17. Flor. Dan. t. 1521. Schkur Krypt. t. 37. Rabenh. Krypt. Polypodium cristatum Lin. Polyp. Callipteris Ehrh. Beiträge, wohl anch Wilms und Wirtgens Flora der preussischen Rheinprovinz p. 548. Waldsiimpfe, sumpfige Wiesen, Torfsümpfe, Erlengebüsche, sehr zerstrent im Gebiet, von den Niederungen bis in die Berg - und Voralpengegenden, aber in vielen I,änderstrichen fehlend. Schweiz stellenweise z. B. bei Thun; Rheingegend, Elsass, Baden im Breisgau, Schwarzach; Hagenauer Forst, Kaiserslautern, Offenbach, im Hengster, Werthheim, dann wieder in den Torfgegenden der niederrheinischen Ebena, Siegburg, Cöln, Düsseldorf, Viersen, Cleve, Aachen; aber wie es scheint fehlt die Pfl. in Belgien und Holland, erscheint aber wieder in Westphalen, Hannover, bei Bremen im Lesumer Moor, in Mecklenburg, bei Halle, im Fichtelgebirge, Sachsen im Mildethale, bei Lukau; Schlesien bei Ohlau, Oppeln, Kleinskal im Bunzlauer Kreise; Ungarn in der Zips, im Szecklerlande am Büdos, um Krakan; Salzburg bei Mittersill, Kärnthen bei Fellach; für Oestr., Tyrol und auch für die südlichen Küstenländer zweifelhaft. 24. Juli-August.

5) P. spin ulos um De Cand. Fl. Fr. 2. Koch Syn. p. 978. Kurzstacheliger Punktfarn. Garke D. Fl. VI. p. 478. Aspidium spinulosum Sw. Syn. Filic. Döll rh. Flora p. 17. Milde schles. Krypt. Asp. dilatatum Godr. flor. de Lorr. Nephrodium spinulosum Strempel Filic. Berol. Polypodium cristatum Hudson Angl. edit. I. und der älteren Autoren, aber nicht Lin. Aendert ab: 
a) P. spinulosum $\alpha$. elevatum, Aspidium $\alpha$. elevatum Al. Braun in Döll rh. Flor. p. 17. Aspidium spinulosum Willd. Spec. pl. 5. Sw. Filic. Syn. Engl. Bot. t. 1460. Schkur Krypt. t. 48. Rabenhorst Krypt. p. 324. Polypodium austriacum Jacq. Observat. I. p. 45.

b) P. spinulosum $\beta$. dilatatum Koch Syn. p. 979. Aspidium spinulosum $\beta$. dilatatum Döll rh. Flora p. 18. Aspidium dilatatum Willd. Spec. pl. V. Sw. Syn. Filic. p. 420. Rabenhorst Krypt. Aspidium spinulosum Schkr. Krypt. t. 47. Aspid. spinulosum $\beta$. Hartm. scand. Polystichum multiflorum Roth tent. III. Polypodium tanacetifolium Hoffm. D. Fl. II. 8. Polypodium dilatatum Hoffm. D. FI. II. 7.

Sumpfige Wälder, Gebüsche, feuchter Moorgrund, aus den Niederungen in die Gebirge und Voralpen, zerstreut, aber nicht selten durch das ganze Gebiet. 24. Juli-August.

6) P. rigidum De Cand. Fl. Fr. 2. Koch Syn. 979. Straffer Punktfarn. Schkur 'Krypt. t. 58. Aspidium rigidum Sw. Syn. Filic. Willd. Spec. pl: V. Döll rh. Flora p. 16. Sturm II. h. 11. Polypodium rigidum Hoffm. D. Fl. 2. Polyp. fragrans Vill. Delph. 3. Polyp. Villarsii Bellard appen. ad flor. Pedemont. Polystichum strigosum Roth tent. III. Lastrea rigida Presl. pterid. Aspidium hastulatum Tenor. Nap. IV. V. t. 250, Boissier Espag. II. oder Aspid. nevadense Boissier Eleng. wird von Boissier, Milde botan. Zeitg. 1857. Wilkomm et Lang Prodr. hisp. I. p. 9. zu Aspidium rigidum Swartz gezogen; Kunze Flora 1858. I. wie Bertol. Cryptog. ziehen die Pflanze zu Aspidium aculeatum Sw.

Felsen der Hochgebirge; Voralpen und Alpen, zerstreut durch die Alpenkette. Piemont, Savoyen, Schweiz, Baiern. Nieder - und Oberöstr. Salzburg, Steiermark, Kärnthen, Tyrol, Vorarlberg bis zum Monte Baldo, Görz, Krain, im Venetianischen und im südlichen Siebenbürgen. 24. Juli-August.

7) P. pallidum (Aspidium pallidum Link Filices p. 107). Blasser Punktfarn. Aspidium rigidum Visiani Dalmat. I. p. 38. teste Heuffel Zool. bot. Ver. 1856. Nephrodium pallidum Bory Exp. de Moree. 3. Nach Gren. et Godr. Franc. III. Wilkomm und Iang nur die südliche Form von Polystichum 
rigidum De Cand. Selten an Felsen in Dalmatien, auf dem Velebit, dem Svilaja, dem Biokoro, bei Clissa, auf der Insel Lesina und in Felsenspalten der Gebirge in Kroatien. 4. Juli - August.

8) P. remotu m Al. Braun. Lockerer Punktfarn. Wirtgen rheinische Reiseflora. I. p. 325. II. p. 165. Aspidium rigidum c. remotnm Döll rh. Flora. p. 16. Koch Syn. p. 979. Polystichum Filix mas c. remotum A]. Braun. Gark e D. Flor. VI. p. 478. Schattige Felsen, früher am Gerolsauer Wasserfall bei Baden, jetzt auch von Al. Braun bei Aachen aufgefunden und wahrscheinlich noch weiter verbreitet. 24. Sommer.

Anmerkung. Nach neueren Beobachtungen von Al. Braun bei Aachen findet sich diese Pflanze nur unter Polystichum Filix mas Roth, ohne die Gegenwart von P. spinulosum D. C. und kann demnach nicht als eine hybride Form von diesen Beiden angesehen werden.

18) Cystopteris Bernh. Schrader neues Journal V. 1. Blasenfarn. Koch Syn. p. 979. Döll rh. Flora p. 14.

1) C. fragilis Bernhardi in Schraders neuem Journale. Zerbrechlicher Blasenfarn. Koch Syn. p. 980. Döll rh. Flora und Flora Badens. I. Cyathea fragilis Bernh. in Schraders Journal von 1792, Roth tent. Fl. germ. III. p. 94. Polypodium fragile Lin., Aspidium fragile Sw. Syn. Filic. D. Cand. Fl. Fr. II. Pollini Ver. III. Sturm II. h. 11. Schkur Krypt. t. 54-56. Aspidium dentatum Sw. Willd. Spec. Polypodium dentatum Dicks. Hoffm. D. Fl. 2. Polypodium rhaeticum Dicks. und vieler älteren Aut. (nicht Lin.). Cyathea regia Roth tent. (nicht Sm. Engl. Bot.). Cyathea fragilis et dentata Smith Brit. Aspidium rhaeticum Willd. Spec. V. Polypodium Pontederae Allion Pedem. Cystopteris fragilis Koch Syn. p. 980. Var. $\alpha$ et $\beta$. Schattige, steinige Stellen in Felsspalten, Schluchten und auf Baumwurzeln der Hügel-, Berg - und Voralpen-Gegenden durch das ganze Gebiet. 24. Juli-August.

2) C. alpina Link Berol. II. p. 130. Alpen-Blasenfarn. Rabenhorst Krypt. p. 319. Aspidium alpinum Sw. Filic. p. 42. Willd. Spec. V. Sturm II, h. 11. Schkur Krypt. t. 62. Cystopteris regia $\beta$. alpina Koch Syn. p. 980. Aendert ab: 
$\beta)$ C. alpina $\beta$ regi a Bernh. Cystopteris regia $\alpha$. fumariaefolia K. Syn. p. 980. Polypodium regium Lin. Spec. Polypodium alpinum Wulf in Jacq. Collect. II. Cyathea incisa Engl. Bot. t. 163. Sm. Brit. (nicht Roth). Aspidium regium Sw. Syn. Filic. Willd. Spec. pl. V. Mit Gebüsche bewachsene Felsen der Alpen and Voralpen, durch die Alpenketten zerstrent.

Italien, Savoyen, Schweiz, Baiern, durch Nieder-Oestr. bis zur Adria nach Dalmatien und Venetien; Tyrol, Vorarlberg, Bozen, Meran bis zum Baldo; dann auf den Karpathen in Ungarn und im südl. Siebenbürgen. 24. August-Septbr.

3) C. monta n a Link hort. bot. Berol. II. Berg-Blasenfarn. Koch Syn. p. 981. Döll rh. Flora. p. 15. Rabenhorst Krypt. p. 319. Polypodium montanum Lamark Frane. I. Hänke in Jacq. Collect. Polypodium myrrhidifolium Vill. Delph. III. Aspidium montanum Sw. Syn. Filic. Fl. Dan. t. 2250. Schkur Krypt. t. 63. Cyathea montana Roth tent. Fl. Germ. An Felsen und steinigen mit Gebüschen bewachsenen Stellen der Alpen und Voralpen durch den grösseren Theil der Alpenkette des Gebietes: Piemont, Savoyen, Schweiz, besonders im Jura und den Appenzeller-Alpen etc. Oestr. Salzburg, Tyrol, Vorarlberg, auf dem Frechen und stellenw. bis zum Baldo, Kärnthen, Krain, Kroatien; dann suf den Karpathen in Galizien, auf der Tatra und am Rodna in Siebenbürgen. 24. AugustSeptember.

4) C. sudetica Alex. Braun und Milde, Schles. Gesellschaft 1855. Milde schles. Krypt. fig. 108 u. 109. Sudeten-Blasenfarn. Garke D. Fl. VI. p. 478. Cystopteris Brannii Milde, C. alpina Wimmer Schles. II. p. 505. C. montana Wimmer, Milde, (nicht Fries, noch Link). C. leucospora (auch leucosoria). Schur Oestr. bot. Zeitg. 1858 u. 1861. Hochgebirgswälder und Waldschluchten an Felsen der Sudeten, im Riesengebirge, im Mährischen Gesenke vom Altvater nach Waldenburg, anf der Hirschwiese und bei Reiwiesen am Hockschar über Ober- und Nieder-Lindewiese; dann in den Karpathen der Tatra in Ungarn und Galizien, dem Bicharia - 
Gebirge im südlichen Ungarn und bei Borszeck im Szecklerlande. 24. Juli-August.

19) Asplenium Lin. Spec. 1178. (excl. spec.) Koch Syn. p. 981. Streifenfarn und Milzfarn.

Heufler Asplenii spec. europaeae Oestr. zool. bot. Ver. 1856. Neilr. Nachträge p. 331.

1) A. Trichomanes Lin. Hudson Angl. edit. I. Brauner Streifenfarn. Koch Syn. p. 982. Rabenhorst Krypt. Garke D. Fl. VI. p. 479. Döll rh. Flora p. 12. Schkur Krypt. t. 74. Mauern, Felsenspalten, Baumwurzeln, durch das ganze Gebiet zerstreut. 4. Juli-- September.

2) A. viride Huds. Angl. edit. II. Grüner Streifenfarn. Koch Syn. Döll rh. Flora p. 13. Garke D. Fl. VI. Rabenhorst Krypt. p. 318. Schkur Krypt. t. 73. Sturm II. h. 1. A. intormedium Presl. In Felsenspalten der Hochgebirge, Voralpen und Alpen, zerstreut idurch die ganze Alpenkette des Gebiets. Piemont, Savoyen, Schweiz, auch auf dem Jura, Oberbaden am Hirschssprung im Höllenthalc, auf Granit und Sandstein in den Vogesen auf dem Rossberge und bei Ribeauviller im Elsass; Westphalen am Wasserfalle bei Ramsbeck, Hannover bei Bittenfeld an Golpaer-Mühle, Harz in Bodethal bei Rübeland; Thüringer Wald am Domberge; Fl. v. Halle, im Fichtelgebirge, Sachsen im Plauenschen Grunde bei Tharand, Sächs. Schweiz am Kuhstall, Baiern auch an den Abhängen der Isar bei München; Schlesien im Riesengebirge im Ruibezahlsgarten, in der kleinen Schneegrube; im Mährischen Gesenke im grossen Kessel; Oestr. bei Wien am Guttenstein und stellenweise in allen Provinzen der Alpen, der Sudeten und Karpathen. 24. Juli-August.

3) A. Filix femina Bernh. in Schraders Journal 1806. Koch Syn. p. 981. Weiblicher Streifenfarn. Garke D. Fl. VI. p. 479. Rabenhorst Krypt. Polypodium Filix femina Lin. Aspidium Filix femina Swartz Filic. Döll Flor. badens. I. Schkar Krypt. t. 58 u. 59. Athyrium Filix femina Roth tent. Athyrium trifidum et Athyrium Molliusculum Baumgarten Transylvan. IV. Aendert in der Form und der Zertheilung der Blattwedel vielfach ab. In Laub- und Nadelholzwäldern, 
an schattigen, quelligen Stellen und an Wiesengräben, nicht selten durch das ganze Gebiet bis in die Berg - und AlpenGegenden. 24. Juli, August und September.

4).A. alpestre Metten. Senkenbergische Gesellschaft 1859. Gebirgs-Streifenfarn. Garke; Aspidium alpestre Hoppe Taschenbuch 1805. Polypodium alpestre Hoppe pl. exsiccat. Koch Syn. p. 974. Spenner fl. Friburg. Wallr. fl. Cryptogam. Rabenhorst Krypt. p. 311. Döll rh. Flora p. 6. Polypod. rhaeticum D. Cand. fl. Franc. V. Villar. Voyag. Schkur Krypt. t. 60. (ob Linné?). Polyp. molle All. Pedem. IV. Phegopteris alpestris Mett. Im äusseren Ansehen ist die Pflanze der vorigen Art sehr ähnlich, die Fruchthäufchen sind auch nur in der Jugend mit einem kleinen Schleierchen versehen, vielleicht Var. von Aspl. Filix femina Bernh.? In Wäldern, Holzschlägen der Hochgebirge, Voralpen und Alpen bis in die Krummholzregion, wohl durch die ganze Alpenkette, wie es scheint, oft nur übersehen. Italien, Piemont, Savoyen Schweiz, Baden, äuf dem hohen Schwarzwalde, auf dem Kandel, dem Feldberg, auf den Hornesgründen, Elsass auf dem Rotabac, dem Ballon von Sültz und Gebweiler; Harz auf dem Brocken und an den Felsen des Bode- und Ocker-Thales, im Erzgebirge in der Nähe des Fichtelberges von Joachimsthal nach Gottesgabe; im Riesengebirge und an den Kämmen des Mährischen Gesenkes, auf den schles. Karpathen, auf der Tatra, den Karpathen des Comit. Saros und des südl. und östl. Siebenbürgen, dann in Niederöstr., Steiermark, Salzburg. 4. Juli - August.

5) A. fontanum Bernh. in Schraders Journal (1799). Quellen-Streifenfarn. A. Halleri D. Cand. fl. Franc. V. Rob. Brow. Koch Syn. p. 982. Aspidium fontanum et Asp. Halleri Willd. Spec. V. Schkur Krypt. t. 53. Polypodium fontanum Lin. herb. nach Sm. Engl. Bot. t. 2024. Polyp. pedicularifolium Hoffm. D. Fl. II. p. 10.

Quellige, feuchte Stellen der Hochgebirge und Alpen, selten im Gebiet. Schweiz, an feuchten Kalkfelsen im Jura, Genf am Saleve, dann im Cant. Tessin; bei Rottenmann in Steiermark, Heiligenblut in Kärnthen; in den Karpathenlän- 
dern, Ungarn bei Rösing Comit. Pest, Siebenbürgen bei Kronstadt und Rodna. 24. Juli - September.

Anmerkung. Die Pflanze soll früher am Lahnberge und bei Marburg nach Garke D. Fl. VI. p. 480, gesammelt worden sein, aber nach Döll rh. Flora p. 12. scheint die Sache auf einer Verwechselung beruhet zu haben, da auch in nenerer Zeit kein rheinischer Florist der Pflanze erwähnt.

6) A. lanceolatum Hudson Angl. edit. II. Lanzettlicher Streifenfarn. A. Billotii Schultz Fl. der Pfalz. A. lanceolatum Döll rh. Flora p. 11. Wirtgen rheinische Reiseflora. Auf Vogesen-Sandstein im Elsass zwischen Weissenburg und Bitsch; zwischen Fischbach und Steinebach in der Gegend von Zweibrïicken in der Baierischen Pfalz. 4. Juli -- August.

7) A. fissum Kitaibel in Willd. Spec. pl. V. Spaltstreifenfarn. Koch Syn. p. 983. Rabenhorst Krypt. p. 315. Heufl. Aspl. lepidum Presl. A. Breynii Pollini Veron. (nicht Retz). A. Trettenerianum Jan. Flora 1859. Aspidium cuneatum Schkur Krypt. t. 56 b. Aspidium tenuifolium Guss. pl. rar. t. 65. Felsen der Alpen und Voralpen, stellenweise im Gebiet. Italien, Oberöstr. auf dem Trauenstein; Niederöstr. auf der Eslingalpe und dem Oetscher, in Steiermark bei Marienzell und auf der Eisenerzer Höhe, Südtyrol im Val di Ronchi, Vallarsa, Valsugana, Roveredo; Krain auf dem Loibl, Recoaro in Vicenza, in Kroatien und Dalmatien, auf der Plisivica, dem Velebit, dem Dinara und den Karpathen in Banat. 24. August - September.

8) A. germanicum Weis Krypt. p. 299. Deutscher Streifenfarn. Garke D. Fl. VI. p. 480. Rabenhorst Krypt. p. 315. Sturm II. h. 5. Aspl. Breynii Retz. Koch Syn. p. 982. Schkur Krypt. t. 81. Aspl. alternifolium Wulf. in Jacq. Misc. II. t. 5, fig. 2. Schattige, etwas feuchte Felsen, Abhänge, Felsenspalten, zerstreut im Gebiete, besonders in Urgebirgsgegenden bis in die Voralpenregion, seltener in den Ebenen. Piemont, Schweiz, von dort durch den südlichen und mittleren Theil der Rheingebirge bis zum Siebengebirge bei Bonn, in Baden im Elsass, in Nassau etc. An der Mosel bei 
Trier, bei Siereck, Luxemburg, Verviers, Eupen bei Aachen; Westphalen, Mecklenburg, Halberstadt, Brandenburg bei Potsdam, Harz, Fichtelgebirge, Sachsen am Königstein, im Plauenschen Grunde, in den Sudeten und Karpathen-Ländern, in Nieder - und Ober-Oestreich, Salzburg, Kärnthen, Tyrol im Oberinnthale, Valsugana, Roveredo, aber nicht in Kroatien and Dalmatien. 24. Juli-September.

9) A. Selosii Leybold. Flora 1853. I. p. 81. t. 15. v. Hausm. Oestr. bot. W. Bl. 1855. Bertol. Krypt. p. 66. Südtyrol. Felsen des Schlerngebirges bei Salurn, in Ampezzo. 24. Sommer.

10) A. Ruta muraria Lin. Spec. Koch Syn. p. 983. Mauerraute. Garke D. Fl. VI. p. 480. Döll rh. Flora p. 10. Rabenhorst Krypt. Schkur Krypt. t. 80 b. A. multicaule Presl. Felsen, Mauern durch das ganze Gebiet bis in die Gebirge, gemein. 24. Juli-September.

11) A. Adiantum nigrum Lin. Spec. Schwarzer Streifenfarn. Koch Syn. Garke D. Fl. p. 480. Döll rh. Flora p. 11. Pollini Veron. III. Heufl. Krypt. p. 300. Aendert ab:

a) nig ru m Heufl. (Binnenlandform). A: Adiantum nigrum Lin. Engl. Bot. t. 1950. Fl. Dan. t. 250. Sturm II. h. 5. Sehkur Krypt. t. 80a. Milde schles. Krypt. fig. 111. bis 113 und 116. A. trichomanoides Lumn. Poson. nach Sadler. A. obtusum Kitaib. in Willd. Spec. pl. V. A. argutum Kaulfuss p. 176. A. silesiacum Milde.

b) On.opteris Heufl. (Südliche Küstenform). A. Onopteris Lin. Spec. A. acutum Bory in Willd. Spec. pl. V. Pollini Ver. t. 2. v. Hausmann zool. bot. Gesell. 1858. II. A. davallioides Tausch Flora 1839. II. A. Virgilii Bory Exp. de Morée III.

c) Serpentini Heufl. Krypt. p. 315. t. 12. (Serpentingebirgsform). A. Serpentini Tausch Flora 1839. A. Serpentini $\beta$. incisum Tausch. A. Adiantum nigrum Schkur Krypt. t. 80 b. A. cuneifolium Vivian. Fragm. I. t. 18 . A. novum Sadl. A. Forsteri Sadl. Filic. Milde schles. Krypt. fig. 105-107. A. incisum Opitz. A. fissam Wimm. Schles. Flora II. (nicht Kitaib.). 
Felsenspalten, etwas schattige, steinige Stellen, in niederen und gebirgigen Gegenden, am verbreites ten im südlichen und westlichen Gebiete, besonders an der Südseite der Ajpen. Italien, Savoyen, Südschweiz, Vorarlberg, Südtyrol bis zum Baldo, Steiermark, Görz; V a r. a. Zerstreut durch das Mittelgebiet, besonder's im Weststrich der rheinischen Gebirge, in Baden, auf Vogesensandstein im Elsass, Porphyr und Trappgebirge am Donnersberg in der Pfalz, an der Nalie, an der Obermosel bei Trier, Luxemburg nach Belgien, bis zum Petersberg bei Mastrich in den Niederlanden, dann wieder bei Heidelberg, Nassau am Taunuszuge und im Lahnthale, Coblenz, auch im Moselgebirge etc., Siebengebirge und Finkenberg bei Bonn; im Harz und mit V a r. c. im Fichtelgebirge, Erzgebirge, in Schlesien, Böhmen, Mähren, in den Sudeten und Karpathen-Ländern, Ungarn, Banat, Siebenbürgen etc. die Var. c. Serpentini besonders in Schlesien am Geiersberg, bei Frankenstein, im Erzgebirge bei Chemnitz, in Böhmen und Mähren. Var. b. die südliche Küstenform am adriatischen Meere in Kroatien, Dalmatien, Venedig und am Mittelmeere. 24. Juli-August.

12) A. septentrionale Hoffm. Deutschl. Fl. II. Nördlicher Streifenfarn. Swartz Filic. Koch Syn. p. 983. Döll rh. Flora p. 8. Garke D. Fl. p. 480. Rabenhorst Krypt. p. 314. Sturm II. h. 5. Schkur Kryptogam. t. 65. Acrostichum septentrionale Linn., Scolopendrium septentrion. Roth tent. $f$. Germ., Acropteris septentrionalis Link. Felsenspalten, Gebirgsabhänge, Mauern, meistens in Berggegenden, geltener in den Ebenen, zerstrent im südlichen und Mittelgebiet, besonders im mittleren Deutschland. Italien, Savoyen, Schweiz, Rheingegend stellenw. bis in die Niederlande, Westphalen, Mecklenburg, Insel Rügen, Brandenburg, Strassburg bei Potsdam, Schlesien im Riesengebirge, Sachsen, Baiern, Würtemberg; Oestr. in allen Provinzen, auf den Alpen, in den Sudeten und Karpathen. 24. Juli-August.

20) Scolopendrium Smith act. taurin. 5. Hirschzunge. Kocb Syn. p. 984. Düll rh. Flora p. 13. 
1) S. officinarum Sw. Filic. (1800). Gebräuchliche Hirschzunge. Döll rh. Fl. Koch Syn. Rabenhorst Krypt. p. 318. Schkur Krypt. t. 83. Scolopendrium vulgare Symens Synop. (1798), Garke D. Flora VI. p. 481. Smith Brit. III. Schattige Bergschluchten, Bergwälder, Abhänge, an inneren Brunnenmauern, zerstreut durch das Gebiet, am häufigsten im südlichen und mittleren Gebiet bis in die Voralpen. 24. JuliAngust.

21) Ble chnum Linn. gen. Rippenfarn. Koch Syn. p. 984. Döll rh. Flora p. 7 .

1) Bl. Spicant Roth tent. III. Withering. Gemeiner Rippenfarn. Koch Syn. p. 984. Döll rh. Flora. Garke D. Fl. VI. p. 481. Rabenhorst Krypt. p. 314. Blechn. boreale Sw. Syn. Filic. Sturm II. h. 11. Schkur Kryptog. t. 110. Acrostichum Spicant Vill. Delph. Both tent. I. Lomaria Spicant Desveaux, Onoclea Spicant Hoffm. D. Fl. 2. Osmunda Spicant Linn., Strutiopteris Spicant Scopoli carn. 2. Schattige, feuchte etwas torfhaltige Wälder, an Felsen und auf Baumwurzeln der Gebirgs - und Voralpengegenden, zerstreut durch das Gebiet. 24. Juli-September.

22) Pteris Linn. 'gener. Saumfarn. Koch Syn. p. 984. Döll rh. Flora p. 7.

1) Pt. aquilina Linn. Spec. Adler-Saumfarn. Koch Syn. Döll rh. Flora. Garke D. Fl. p. 481. Rabenhorst Krypt. Sturm II. h. 1. Schkur Krypt. t. 95. Pt. brevipes Tausch Flora 1836. II. eine Form mit kurzgestielten Wedeln. Var. Pt. aquilina $\beta$. lanuginosa Hocker. Wälder, Waldwiesen, Holzschläge, Heiden bis in die Voralpen, durch das ganze Gebiet. 4. Juli-September.

2) Pt. cre tica Linn. Spec. Kretischer Saumfarn. Spreng. Syst. Vol. IV Pt. semiserrata Forsk. Pt. heptaphyllos Poir. Im südlichsten Gebiet, in den Mittelmeergegenden bei Nizza, Piemont bei Tender und in der italienischen Schweiz im Cant. Tessin. 24. Sommer.

23) Cheilanthes Swartz Syn. Filic. Spreng. Syst. IV. Cheilanthie. 
Uebersicht der Gefäss - Kryptogamen, Farnkräuter etc.

1) Cheil. odora Sw. Syn. Filic. p. 127 und 327. Rabenhorst Kryptog. p. 320. Schkur Krypt. t. 123. Cheil. suaveolens Sw. Filic. p. 127. Flora gr. X. t. 966. Cheil. fragrans Hocker, Webb. et Berth. Canar. III. (nicht Cheil. fragrans Sw. Syn. p. 127. t. 3, welches eine Pflanze Indiens) Polypodium fragrans Linn. Mant. II. p. 307. Desfont. Atlan. II. t. 257. Adiantum fragrans Lamark et D. Cand. Syn. pl. in flor. Gall. p. 112. Felsen am südlichen Litoral, in Dalmatien um Cattaro bei St. 'Trinita, um Ragusa, auf Lissa, Giuppana und Lesina; am Mittelmeere bei Oneglia, um Nizza; dann in Oberitalien bei Turin, in der italienischen Schweiz im AostaThale zwischen Domo d'Ossola und Villa, zweifelhaft für Tessin. 24. Juli-August.

2) Cheil. Scovitsii Fisch. et Meyer Bull. soc. Mosc. 1838. p. 241. Ledeb. Ross. IV. Neilr. Nachtr. p. 334. Cheil. fimbriata Visiani fl. Dalmat. I. 1842. p. 42. III. p. 335. nach Kunze Bot. Zeit. 1844. p. 277. Acrostichum microphyllum Bèrtoloni Crypt. p. 35. v. Hausmann zool. bot. Gesell. 1860. p. 71. Südtyrol an Felsen des Monte Baldo, Dalmatien auf den Inseln Lesina und Giuppana und wohl auch am Küstengebirge des Mittelmeeres. 24. Sommer.

24) A diantum Linn. gen. Haarfarn, Lappenfarn. Koch Syn. p. 984. Döll rh. Flora. p. 6.

1) A. Capillus veneris Linn. Spec. Jacq. Misc. П. t. 7. Frauenhaar. Koch Syn. Döll rh. Fl. Rabenhorst Krypt. p. 309. Engl. Bot. t. 1564. Feuchte Felsen, Bergschluchten im südlichen Gebiet, selten dieseits der Alpen; Italien, Schweiz in den Tuffstein-Grotten bei Aubin am Neuchateller See und durch den Jura bis zum Chasseral; in Südtyrol am GardaSee etc. In Innerkrain bei Idria, Görz, Triest, Istrien, Kroatien, Dalmatien, Venedig und im Comit. Hunyad in Siebenbürgen. 24. Juli - September.

25) Nothochlaena Rob. Brow. prodr. nov. Holland. edit. Nees ab Esenbeck. Nothochläne. Koch Syn. p. 985.

1) N. Marantae Rob. Brown. Koch Syn. Rabenhorst Krypt. p. 312. Acrostichum Marantae Linn. Spec. Sturm II. h. 6. Schkur Krypt. t. 4. Ceterach Marantae De Cand. fl. 
franc. I. Gymnogramma Marantae Mett. Felsen des Bergund Hügellandes im südlichen Gebiete. Südschweiz im Canton Tessin, Südtyrol im Vintschgan, bei Bozen, Brixen, Meran und auf den Euganeen; Ungarn bei Agram; in Syrmien; dann auch in Niederöstr. im Gurhofgraber und im Znaimer Kreise bei Mohelno. 4. Juli-August.

26) Allosorus Bernhardi in Schraders Journ. 1806. Koch Syn. 985. Rollfarn.

1) A. crispus Bernh. Koch Syn. Krauser Rollfarn. Garke D. Fl. p. 481. Cryptogramma crispa. Rob. Brow. Döll rh. Flora p. 3. Rabenh. Krypt. p. 312. Pteris crispa Allion. Pedem. II. p. 284. Schkur Kryptogam. t. 98. Onoclea crispa Hoffm. D. Fl. I. Sturm II. h. 1. Osmunda crispa Lin. Spec. Acrostichum crispum Vill. Delph. 4. p. 838. Phorolobus crispus Desv., Struthiopteris crispa Wallr. in Comp. flor. german. III. p. 27. Felsenspalten und auf steinigen Triften der Hochgebirge, Voralpen und Alpen stellenweise, doch selten, durch die Gebirgsketten. Italien, Piemont, Savoyen, Schweiz, Rheingegend in Oberbaden auf dem hohen Schwarzwalde, auf dem Schauinsland beim Hofgrund nicht fern von Freiburg im Breisgau; Elsass auf den Vogesen, Ballon von Gebweiler, Sulz, Hoheneck und auf dem Rotabac etc. Dann am Harz bei Goslar; Schlesien im Riesengebirge, in der Schneegrube am Teufelsgärtchen und im Riesengrunde, an den Abhängen des Brunnenberges und auf den Karpathen des Comit. Hunyad in Siebenbürgen; dann auf den Alpen in Oestr., im Böhmerwalde, auf dem Wechsel in Niederöstr., Salzburg, Obersteiermark, Tyrol im Oetzthale gegen Tend, am Passeyer, Sarnthal und in den Alpen Baierns. 24. Juli-September. Laub dem von Asplenium Ruta muraria Linn ähnlich.

27) Struthiopteris Willd. Enum. hort. Berol. II. Straussenfarn. Koch Syn. p. 983. Döll rh. Fl. p. 21.

1) St. germanica Willd. Berolin. II. Deutscher Straussenfarn. Döll rh. Fl. Garke D. Fl. p. 482. Rabenhorst Krypt. p. 325. Milde Schles. Krypt. fig. 65-80. Onoclea Struthiopteris Hoffm. D. Fl. II. Schkur Krypt. t. 105. Osmunda Struthiopteris Linné. An steinigen, etwas feuchten und schat- 
tigen Stellen, in Bergwäldern, an Waldbächen und auf etwas sumpfigen Wiesen, sehr zerstreut im Gebiete bis in die Voralpengegenden, ist aber in vielen Ländern des Gebiets, wie es scheint, noch nicht aufgefunden worden; z. B. auf der Siidseite der Alpen, dann in den südlichen und nördlichen $K \ddot{u}$-. stenstrichen, wie auch in Holland, Hannover, Mecklenburg etc. und ist auch für die Schweiz sehr zweifelhaft.

Dieser schöne Farn findet sich in der Rheingegend in Oberbaden bei Oberkirch, bei Oppenau, bei dem Bade Antogast und am Murgufer bei Gaggenau; in der Wetterau am Feldberg, in Nassau bei Reifenberg und Usingen und am Kaltenbachthale bei Homburg; dann rheinabwärts im Wiedbachthale bei Arensau, im Siegthale bei Kirchen und bei Siegburg, im Aggerthale bei Lomar Fl. v. Bonn und an der Wupper bei Neuenkirchen, am Fusse des Hülsenstein Fl. v. Cöln, scheint aber im Weststriche der Rheingegend zu fehlen und ist für diesen nur auf der Grenze der Fl. v. Aachen um Fays bei Verviers angegeben; dann kommt die Pflanze wieder am Harz, Halle bei Schmon, am Giebichenstein, bei Dessau, Preussen bei Soran, Sachsen am Hohenstein bei Chemnitz und wohl weiter verbreitet vor; Schlesien bei Laasan im Briesnitzgrund, bei Naumburg an der Bober, Halban, an der Weichsel bei Ustron und stellenweise bis zur Oberlausitz; dann in Böhmen bei Stirim im Prager Kreise, in den Karpathen-Thïlern, in Böhmen, Mähren, Galizien, Ungarn und im südlichen und östlichen Siebenbürgen; in den Voralpen, im nördlichen Tyrol im Zillerthale am Grimmenberg, in Kärnthen, Salzburg, Steiermark, Ober - und Niederöstreich etc. 24. JuliSeptember.

Nota. Hauptsächliche Werke, welche bei obiger Zusammenstellung benutzt wurden:

Bertoloni Flora italica cryptogama. Bononiae 1858. Bertol. Crypt.

Döll Rheinische Flora. Frankf. a/M. 1843. Döll rh. Flora.

Garke Flora von Nord- und Mittel-Deutschl. VI. Aufl. Berlin 1863. Garke D. FI. VI. 
Uebersicht der Gefäss - Kryptogamen, Farnkräuter etc.

Gremli Excursionen-Flora der Schweiz. Aarau 1867. Gremli Exc. Fl. d. Schweiz.

Koch Synopsis Fl. Germ. et Helv. edit. 2. 1843. Koch Syn.

Milde Die Gefäss-Kryptogamen Schlesiens. Breslau und Bonn 1858. Milde schles. Krypt.

Rabenhorst Deutschlands Kryptogamen-Flora. Leipzig 1848. Rabenh. Krypt.

Neilreich Nachträge zu Malys Enumerat. Wien 1861. Anhang p. 321. Neilr. Nachtr.

Schkur 24. Class. des Linnéischen Pflanzensystems, oder die kryptogamischen Gewächse. Wittenberg 1809. Schkr. Kryptogamen.

Sturm Deutschlands Flora in Abbildungen nach der Natur. II. Abtheilung, Kryptogamen. Nürnberg 17981833. 25 Hefte. Sturm II. h.

Swartz Filices. Kiliae 1806. Cöln a/R. Sw. Filic. 1868. 